\title{
Ricci-flat graphs with girth at least five
}

\author{
Yong Lin, LinYUAN LU AND S.-T. YAU
}

\begin{abstract}
A graph is called Ricci-flat if its Ricci curvatures vanish on all edges. Here we use the definition of Ricci curvature on graphs given in Lin-Lu-Yau, Tohoku Math., 2011, which is a variation of Ollivier, J. Funct. Math., 2009. In this paper, we classified all Ricci-flat connected graphs with girth at least five: they are the infinite path, cycle $C_{n}(n \geq 6)$, the dodecahedral graph, the Petersen graph and the half-dodecahedral graph. We also construct many Ricci-flat graphs with girth 3 or 4 by using the root systems of simple Lie algebras.
\end{abstract}

\section{Introduction}

The Ricci curvature plays a very important role in geometric analysis on Riemannian manifolds. Ricci-flat manifolds are Riemannian manifolds with Ricci curvature vanishes. In physics, they represent vacuum solutions to the analogs of Einstein's equations for Riemannian manifolds of any dimension, with vanishing cosmological constant. An important class of Ricci-flat manifolds is Calabi-Yau manifolds. This follows from Yau's proof of the Calabi conjecture, which implies that a compact Kähler manifold with a vanishing first real Chern class has a Kähler metric in the same class with vanishing Ricci curvature. There are many works on constructing the Calabi-Yau manifolds. Yau conjectured that there are finitely many topological types of compact Calabi-Yau manifolds in each dimension. This conjecture is still open. In this paper, we will study this question on graphs. First we will give a short history of the definition of Ricci curvature in discrete setting.

The definition of the Ricci curvature on metric spaces was first from the well-known Bakry and Emery notation. Bakry and Emery [1] found a way to define the "lower Ricci curvature bound" through the heat semigroup $\left(P_{t}\right)_{t \geq 0}$ on a metric measure space $M$. There are some recent works on giving a good notion for a metric measure space to have a "lower Ricci curvature bound," see $[17,18,20]$. Those notations of Ricci curvature work on so-called length spaces. In 2009, Ollivier [19] gave a notion of coarse Ricci curvature of Markov chains valid on arbitrary metric spaces, such as graphs. 
Graphs and manifolds are quite different in their nature. But they do share some similar properties through Laplace operators, heat kernels, random walks, etc. Many pioneering works were done by Chung, Yau and their coauthors [2-13].

A graph $G=(V, E)$ is a pair of the vertex-set $V$ and the edge-set $E$. Each edge is an unordered pair of two vertices. Unless otherwise specified, we always assume a graph $G$ is simple (no loops and no multi-edges) and connected. It may have infinite but countable number of vertices. For each vertex $v$, the degree $d_{v}$ is always finite. Starting from a vertex $v_{1}$ we select a vertex $v_{2}$ in the neighborhood of $v_{1}$ at random and move to $v_{2}$ then we select a vertex $v_{3}$ in the neighborhood of $v_{2}$ at random and move to $v_{3}$, etc. The random sequence of vertices selected this way is a random walk on the graph. Ollivier's [19] definition of the coarse Ricci curvature of Markov chains on metric space can be naturally defined over such graphs.

The first definition of Ricci curvature on graphs was introduced by Fan Chung and Yau [5] in 1996. In the course of obtaining a good log-Sobolev inequality, they found the following definition of Ricci curvature to be useful.

We say that a regular graph $G$ has a local $k$-frame at a vertex $x$ if there exist injective mappings $\eta_{1}, \ldots, \eta_{k}$ from a neighborhood of $x$ into $V$ so that

(1) $x$ is adjacent to $\eta_{i} x$ for $1 \leq i \leq k$ and

(2) $\eta_{i} x \neq \eta_{j} x$ if $i \neq j$.

The graph $G$ is said to be Ricci-flat at $x$ if there is a local $k$-frame in a neighborhood of $x$ so that for all $i$

$$
\bigcup_{j}\left(\eta_{i} \eta_{j}\right) x=\bigcup_{j}\left(\eta_{j} \eta_{i}\right) x .
$$

For a more general definition of Ricci curvature, in [16], Lin and Yau give a generalization of lower Ricci curvature bound in the framework of graphs in term the notation of Bakry and Emery.

In our previous paper [15], the Ricci curvature on graphs is defined based on Ollivier's definition of Ricci curvature for Markov chains on graphs. It is natural to define a Ricci-flat graph to be a graph where Ricci curvature vanishes on every edge. This definition does not require a graph to be regular; which is an advantage over the Chung-Yau's definition. The Ricci-flat graphs defined by Chung and Yau are not necessarily Ricci-flat in the sense of our definition. However, the Ricci curvatures of those graphs are always nonnegative. In the last section, we constructed many "Ricci-flat" graphs under both definitions. 
A well-known Bonnet-Myers theorem on Riemannian geometry said that if a complete Riemannian manifolds with Ricci curvature bounded below by a positive constant, then it is compact and has a finite fundamental group. In [15], we prove the first part result of Bonnet-Myers theorem on graphs with Ricci curvature bounded below by a positive constant. In the paper of [14], Forman introduced the Ricci curvature on cell complexes and also obtained the Myers theorem on the fundamental group of the complexes.

In this paper, we classified Ricci-flat graphs with large girth (using our definition).

Theorem 1. Suppose that $G$ is a Ricci-flat graph with girth $g(G) \geq 5$. Then $G$ is one of the following graphs:

(1) the infinite path,

(2) cycle $C_{n}$ with $n \geq 6$,

(3) the dodecahedral graph,

(4) the Petersen graph and

(5) the half-dodecahedral graph.

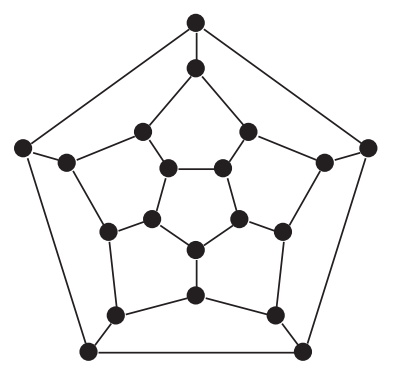

Dodecahedral graph

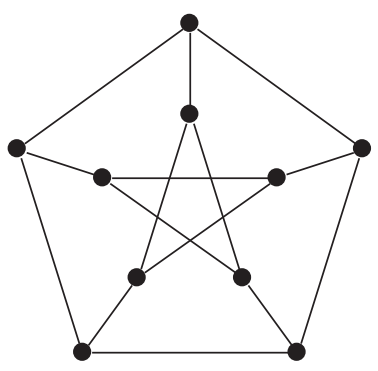

Petersen graph

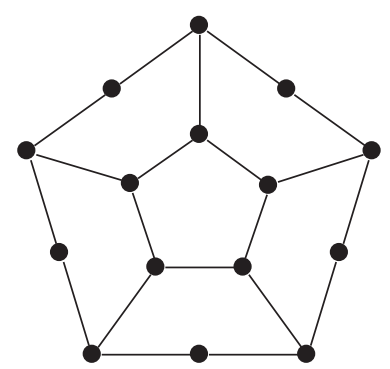

Half-dodecaheral graph

The paper is organized as follows. In Section 2, we will review the definitions and some facts about Ricci curvature on graphs. Theorem 1 will be proved in Section 3. In the last section, we will discuss general constructions using Cartesian product, strong graph covering and lattice graphs constructed by the root systems of simple Lie algebras. Combining these methods, we constructed many Ricci-flat graphs with girth 3 or 4 . 


\section{Notations and lemmas}

Let $G=(V, E)$ be a simple graph. A probability distribution (over the vertex-set $V(G))$ is a mapping $m: V \rightarrow[0,1]$ satisfying $\sum_{x \in V} m(x)=1$. Suppose that two probability distributions $m_{1}$ and $m_{2}$ have finite support. A coupling between $m_{1}$ and $m_{2}$ is a mapping $A: V \times V \rightarrow[0,1]$ with finite support so that

$$
\sum_{y \in V} A(x, y)=m_{1}(x) \quad \text { and } \quad \sum_{x \in V} A(x, y)=m_{2}(y) .
$$

The transportation distance between two probability distributions $m_{1}$ and $m_{2}$ is defined as follows:

$$
W\left(m_{1}, m_{2}\right)=\inf _{A} \sum_{x, y \in V} A(x, y) d(x, y),
$$

where the infimum is taken over all couplings $A$ between $m_{1}$ and $m_{2}$. A function $f$ over $G$ is $c$-Lipschitz if

$$
|f(x)-f(y)| \leq c d(x, y)
$$

for all $x, y \in V$. By the duality theorem of a linear optimization problem, the transportation distance can also be written as follows:

$$
W\left(m_{1}, m_{2}\right)=\sup _{f} \sum_{x \in V} f(x)\left[m_{1}(x)-m_{2}(x)\right],
$$

where the supremum is taken over all one-Lipschitz function $f$.

For any vertex $x$, let $\Gamma(x)$ denote the set of neighbors of $x$, i.e.,

$$
\Gamma(x)=\{v \mid v x \in E(G)\} .
$$

Let $N(x)=\Gamma(x) \cup\{x\}$.

For any $\alpha \in[0,1]$ and any vertex $x$, the probability measure $m_{x}^{\alpha}$ is defined as

$$
m_{x}^{\alpha}(v)= \begin{cases}\alpha & \text { if } v=x \\ \frac{1-\alpha}{d_{x}} & \text { if } v \in \Gamma(x) \\ 0 & \text { otherwise }\end{cases}
$$




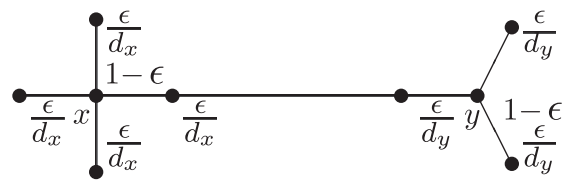

Figure 1: The transportation distance between two $\epsilon$-balls in a graph.

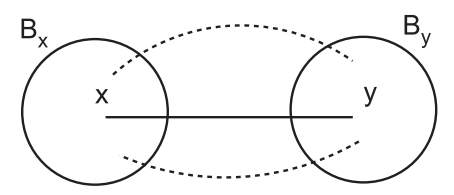

Figure 2: The average distance between two $\epsilon$-balls in a manifold.

For any $x, y \in V$, we define $\alpha$-Ricci curvature $\kappa_{\alpha}$ to be

$$
\kappa_{\alpha}(x, y)=1-\frac{W\left(m_{x}^{\alpha}, m_{y}^{\alpha}\right)}{d(x, y)}
$$

The Ricci curvature $\kappa(x, y)$ is defined as

$$
\lim _{\alpha \rightarrow 1} \frac{\kappa_{\alpha}(x, y)}{1-\alpha}
$$

Let $\epsilon=1-\alpha$. Then $m_{x}^{\alpha}$ can be viewed as the ball of radius $\epsilon$ and centered at $x$. Under this setting, the Ricci curvature captures the approximation of the transportation distance between two balls of radius $\epsilon$ :

$$
W\left(m_{x}^{1-\epsilon}, m_{y}^{1-\epsilon}\right)=(1-\epsilon \kappa(x, y)+o(\epsilon)) d(x, y)
$$

This is similar to the Ricci curvature in the differential manifolds $M$ of dimension $N$ (see figures 1 and 2). Let $x \in M$ and $v$ be a unit tangent vector at $x$. Let $y$ be a point on the geodesic issuing from $v$, with $d(x, y)$ small enough. Then, the average distance between two $\epsilon$-balls centered at $x$ and $y$ in $M$ is (see [19]):

$$
d(x, y)\left(1-\frac{\epsilon^{2} \operatorname{Ric}(v, v)}{2(N+2)}+O\left(\epsilon^{3}+\epsilon^{2} d(x, y)\right)\right)
$$

A graph $G$ is called Ricci-flat if $\kappa(x, y)=0$ for any edge $x y \in E(G)$. 
Lemma 1. Suppose that an edge $x y$ in a graph $G$ is not in any $C_{3}, C_{4}$ or $C_{5}$. Then $\kappa(x, y)=\frac{2}{d_{x}}+\frac{2}{d_{y}}-2$.

Corollary 1. Suppose that $x$ is a leaf-vertex (i.e., $d_{x}=1$ ). Let $y$ be the only neighbor of $x$. Then $\kappa(x, y)>0$.

Lemma 2. Suppose that an edge $x y$ in a graph $G$ is not in any $C_{3}$ or $C_{4}$. Then $\kappa(x, y) \leq \frac{1}{d_{x}}+\frac{2}{d_{y}}-1$.

Proof. Define $f: N(x) \cup N(y) \rightarrow \mathbb{R}$ as follows:

$$
f(u)= \begin{cases}0 & \text { if } u \in N(x) \backslash\{y\}, \\ 1 & \text { if } u=y, \\ 2 & \text { if } u \in N(y) \backslash\{x\} .\end{cases}
$$

Since $x y$ is not in any $C_{3}$ or $C_{4}, f$ is a Lipschitz function. We have

$$
\begin{aligned}
W\left(m_{x}^{\alpha}, m_{y}^{\alpha}\right) & \geq \sum_{u \in V} f(u)\left[m_{y}^{\alpha}(u)-m_{x}^{\alpha}(u)\right] \\
& =\alpha+2(1-\alpha)\left(1-\frac{1}{d_{y}}\right)-\frac{1-\alpha}{d_{x}} \\
& =2-\alpha-(1-\alpha)\left(\frac{1}{d_{x}}+\frac{2}{d_{y}}\right) .
\end{aligned}
$$

We have

$$
\kappa(x, y)=\lim _{\alpha \rightarrow 1} \frac{1-W\left(m_{x}^{\alpha}, m_{y}^{\alpha}\right)}{1-\alpha} \leq \frac{1}{d_{x}}+\frac{2}{d_{y}}-1 .
$$

The proof of the lemma is finished.

Lemma 3. Suppose that an edge $x y$ in a graph $G$ is not in any $C_{3}$ or $C_{4}$. Without loss of generality, we assume $d_{x} \leq d_{y}$. If $\kappa(x, y)=0$, then one of the following statements holds (see figure 3):

(1) $d_{x}=d_{y}=2$. In this case, $x y$ is not in any $C_{5}$.

(2) $d_{x}=d_{y}=3$. In this case, $x y$ is shared by two $C_{5}$ 's.

(3) $d_{x}=2$ and $d_{y}=3$. In this case, let $x_{1}$ be the other neighbor of $x$ other than $y$. Let $y_{1}, y_{2}$ be two neighbors of $y$ other than $x$. Then $\left\{d\left(x_{1}, y_{1}\right), d\left(x_{1}, y_{2}\right)\right\}=\{2,3\}$. 

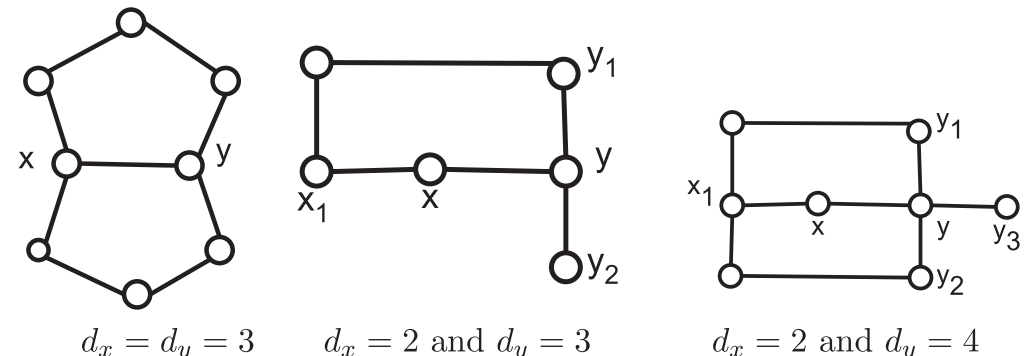

$d_{x}=d_{y}=3$

$$
d_{x}=2 \text { and } d_{y}=3
$$$$
d_{x}=2 \text { and } d_{y}=4
$$

Figure 3: Local structures in Lemma 3.

(4) $d_{x}=2$ and $d_{y}=4$. In this case, let $x_{1}$ be the other neighbor of $x$ other than $y$. Let $y_{1}, y_{2}, y_{3}$ be three neighbors of $y$ other than $x$. Then at least two of $y_{1}, y_{2}, y_{3}$ have distance 2 from $x$.

Proof. By Lemma 2, we have

$$
0 \leq \frac{1}{d_{x}}+\frac{2}{d_{y}}-1
$$

Since $d_{x} \leq d_{y}$, we have $1 \leq \frac{3}{d_{x}}$. By Corollary $1, d_{x} \geq 2$. We have $d_{x}=2$ or 3 . If $d_{x}=2$, then $d_{y}=2,3,4$. If $d_{x}=3$, then $d_{y}=3$.

Case 1. $d_{x}=d_{y}=2$ and $x y$ is in a $C_{5}$, then $\kappa(x, y)>0$. Contradiction!

Case 2. $d_{x}=d_{y}=3$ and $x y$ is not shared by two $C_{5}$ 's. Let $x_{1}, x_{2}$ be two other neighbors of $x$ and $y_{1}, y_{2}$ are two other neighbors of $y$. Without loss of generality, we can assume $d\left(x_{1}, y_{1}\right) \geq 3$ and $d\left(x_{1}, y_{2}\right) \geq 3$. In this case, we can define a Lipschitz function $f: N(x) \cup N(y) \rightarrow \mathbb{R}$ as follows, $f\left(x_{1}\right)=-1$, $f\left(x_{2}\right)=f(x)=0, f(y)=1$ and $f\left(y_{1}\right)=f\left(y_{2}\right)=2$. Using this function $f$, we can show $\kappa(x, y)<0$. Contradiction.

Case 3. $d_{x}=2$ and $d_{y}=3$. Let $x_{1}$ be the other neighbors of $x$ and $y_{1}, y_{2}$ are two other neighbors of $y$. If $d\left(x_{1}, y_{1}\right)=d\left(x_{1}, y_{2}\right)=2$, then a similar calculation shows $\kappa(x, y)>0$. If $d\left(x_{1}, y_{1}\right)=d\left(x_{1}, y_{2}\right)=3$, then $\kappa(x, y)=$ $\frac{2}{2}+\frac{2}{3}-1<0$. In both cases, we get contradiction.

Case $4 \cdot d_{x}=2$ and $d_{y}=4$. Let $x_{1}$ be the other neighbors of $x$ and $y_{1}, y_{2}, y_{3}$ are two other neighbors of $y$. If $d\left(x, y_{i}\right)(i=1,2,3)$ have at least two 3's, then $\kappa(x, y)<0$. Contradiction!

The proof of Lemma is finished. 


\section{Proof of Theorem 1}

Proof of Theorem 1. Suppose $g(G) \geq 6$. By Lemma 1, for any edge $x y$, we have

$$
0=\kappa(x, y)=\frac{2}{d_{x}}+\frac{2}{d_{y}}-2 .
$$

The only integer solution is $d_{x}=d_{y}=2$. Hence $G$ is two-regular graph. Since $G$ is connected, it is either an infinite path or a cycle $C_{n}$.

Now we consider the case $g(G)=5$. By Lemma 3, every vertex in $G$ has degree 2,3 or 4 . We have the following three claims:

Claim a. $G$ has no vertex with degree 4 .

Suppose that $y$ is a vertex with degree 4 . By Lemma 3, all its neighbors must have degree 2 . Let $x$ be one of its neighbors and $x_{1}$ be the other neighbor of $x$ other than $y$. The local structure is shown in figure 3 . We observe that $x_{1}$ have degree at least 3 . If $d_{x_{1}}=3$, by Lemma $3, x_{1} x$ cannot be shared by two $C_{5}$; contradiction! If $d_{x_{1}}=4$, then all its neighbors have degree 2 . Now we have two vertices of degree 2 in a $C_{5}$ that are adjacent to each other. This is a contradiction to item 1 of Lemma 3 . Thus, we proved Claim a.

Claim $b$. Any $C_{5}$ in $G$ can have at most one vertex with degree 2.

Suppose that $u$ and $v$ are two vertices of degree 2 located in one $C_{5}$. By Item 1 of Lemma 3, the vertices with degree 2 are not adjacent to each other. In particular, the other three vertices on this $C_{5}$ have degree 3 . Let $x, y$ be two adjacent vertices of degree 3 on a $C_{5}$. Applying Lemma 3 , there is another $C_{5}$ passing through $x y$. The other vertices are labeled as shown in figure 4.

If $d_{u^{\prime}}=3$, then $x u^{\prime}$ is in two $C_{5}$. As result, $w w^{\prime}$ must be connected. Then $v w$ is in two $C_{5}$ 's. Contradiction to item 3 of Lemma 3 . Hence $d_{u^{\prime}}=2$.

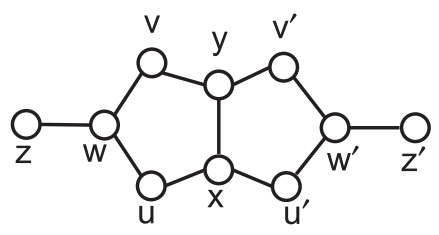

Figure 4: Two $C_{5}$ 's. 
Similarly, we have $d_{v^{\prime}}=2$. Note that $d_{w}=d_{w^{\prime}}=3$. Note $z w$ is not in any $C_{5}$. Contradiction. Claim b is proved.

Claim c. Suppose that $x y$ is an edge of $G$ with $d_{x}=2$ and $d_{y}=3$. Let $y_{1}$ and $y_{2}$ be the other two neighbors of $y$ besides $x$. Then one of $y_{1}$ and $y_{2}$ has degree 2 while the other one has degree 3.

By Lemma 3, every vertex of degree 2 is in a $C_{5}$. This $C_{5}$ must contain $y$ and one of $y_{1}$ and $y_{2}$. Without loss of generality, assume that $y_{1}$ is in this $C_{5}$. By Claim b, $y_{1}$ must have degree 3. By Lemma 3, $y y_{1}$ is shared by two $C_{5}$ 's. The second $C_{5}$ must pass through $y_{2}$. Since $x y$ is only in one $C_{5}$, there is no $C_{5}$ containing $y_{2}, y$ and $x$. Thus, $d_{y_{2}}=2$.

If $G$ has no vertex with degree 2, then $G$ is three-regular. Every edge is in two $C_{5}$ 's. $G$ can be embedded into a surface $S$.

If $S$ is an oriented surface with genus $s$, then Euler formula gives

$$
n-e+f=2-2 s \text {. }
$$

Here $e$ is the number of edges and $f$ is the number of faces. Since $G$ is three-regular, we have $2 e=3 n$. Since every face is a $C_{5}$, we have $2 e=5 f$. Thus

$$
2-2 s=n-e+f=\frac{2}{3} e-e+\frac{2}{5} e=\frac{1}{15} e>0 .
$$

We must $s=0$. Hence $e=30, n=20$ and $f=10$. In this case, $G$ is the dodecahedral graph.

If $S$ is a non-oriented surface with odd genus $s$, then Euler formula gives

$$
n-e+f=2-s
$$

Here $e$ is the number of edges and $f$ is the number of faces. Since $G$ is three-regular, we have $2 e=3 n$. Since every face is a $C_{5}$, we have $2 e=5 f$. Thus

$$
2-s=n-e+f=\frac{2}{3} e-e \frac{2}{5} e=\frac{1}{15} e>0 .
$$

We must $s=1$. Hence $e=15, n=10$ and $f=6$. In this case, $G$ is the Petersen graph.

If $G$ contains a vertex $u$ with degree 2 , then $u$ is in a $C_{5}$. The other four vertices have degree 3 . By Lemma 3 , this forces four $C_{5}$ s as shown by figure 5.

By Claim c, we have $d_{v}=d_{w}=2$. By Claim b, we have $d_{x}=d_{y}=3$. Now the graph continues to expand. It finally results in the half-dodecahedral graph. The proof of theorem is finished. 


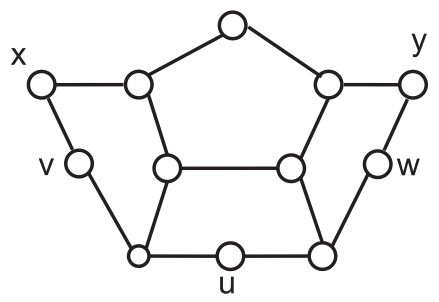

Figure 5: A partial graph of the half-dodecahedral graph.

\section{More Ricci-flat graphs}

In this section, we will construct many Ricci-flat graphs with girth 3 or 4. Given two graphs $G$ and $H$, the Cartesian product (denoted by $G \square H$ ) is a graph over the vertex-set $V(G) \times V(H)$, where two pairs $\left(u_{1}, v_{1}\right)$ and $\left(u_{2}, v_{2}\right)$ are connected if " $u_{1}=u_{2}$ and $v_{1} v_{2} \in E(H)$ " or " $u_{1} u_{2} \in E(G)$ and $v_{1}=v_{2} . "$ The following theorem is proved in [15].

Theorem (see [15]). Suppose that $G$ is $d_{G}$-regular and $H$ is $d_{H}$-regular. Then the Ricci curvature of $G \square H$ is given by

$$
\begin{aligned}
\kappa^{G \square H}\left(\left(u_{1}, v\right),\left(u_{2}, v\right)\right) & =\frac{d_{G}}{d_{G}+d_{H}} \kappa^{G}\left(u_{1}, u_{2}\right), \\
\kappa^{G \square H}\left(\left(u, v_{1}\right),\left(u, v_{2}\right)\right) & =\frac{d_{H}}{d_{G}+d_{H}} \kappa^{H}\left(v_{1}, v_{2}\right) .
\end{aligned}
$$

Here $u \in V(G), v \in V(H), u_{1} u_{2} \in E(G)$ and $v_{1} v_{2} \in E(H)$.

Corollary 2. If both $G$ and $H$ are Ricci-flat regular graphs, so is the Cartesian product graph $G \square H$.

Another construction of Ricci-flat graphs is using a strong graph covering. A graph $G$ is a strong covering graph of another graph $H$ if there is a surjective map $f: V(G) \rightarrow V(H)$ satisfying for any edge $u v \in E(G), f$ maps the induced graph of $G$ on $\Gamma_{G}(u) \cup \Gamma_{G}(v)$ to the induced graph of $H$ on $\Gamma_{H}(f(u)) \cup \Gamma_{H}(f(v))$ bijectively. Note in the traditional definition of covering graph, it only requires $f$ maps the induced graph of $G$ on $\Gamma_{G}(u)$ to $H$ on $\Gamma_{H}(f(u))$ bijectively. A strong covering graph $H$ is always a cover graph of $H$, not vice versa. Observe that $\kappa(u, v)$ only depends on the induced graph on $\Gamma(u) \cup \Gamma(v)$. We have the following corollary. 
Corollary 3. If $G$ is a strong covering graph of $H$, then $G$ is Ricci-flat if and only if $H$ is Ricci-flat.

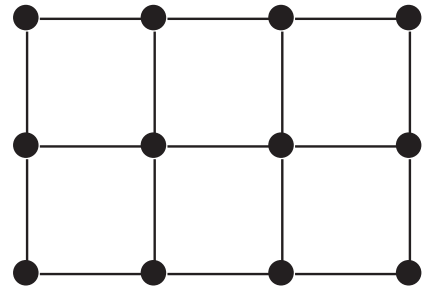

Cartesian Product

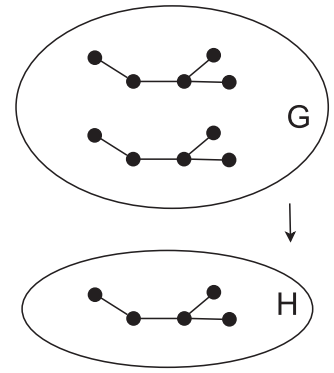

Strong covering graphs

A Ricci-flat graph $G$ is called simple if $G$ is neither the Cartesian product of two Ricci-flat graphs or the projection of a Ricci-flat graph.

Many simple Ricci-flat graphs can be constructed using Cayley graphs. Suppose that $G$ is a group and $S$ is a subset of $G$ with $-S=S$. The Cayley graph Cayley $(G, S)$ is a graph with the vertex set of all elements in $G$ and where a pair $(x, y)$ forms an edge if $x^{-1} y \in S$.

Theorem 2. Let $G$ be a torsion-free abelian group and $S$ be a finite subset of $G$ satisfying $S=-S$. Let $H:=$ Cayley $(G, S)$ be the Cayley graph. Suppose that $H$ has following property "for any $x \in V, s \in S$ and $n \geq 1$, the graph distance $d_{H}(x, x+n s)=n$." Then $H$ is Ricci-flat.

Proof. We first show $\kappa(x, y) \geq 0$ for any edge $x y$. We use addition "+" for the group operation of the abelian $G$. Note that $H$ is vertex-transitive; thus it is regular with degree $d:=|S|$. For $\alpha \in[0,1]$, we need to construct a transportation $A$ that moves the probability distribution $m_{x}^{\alpha}$ to $m_{y}^{\alpha}$ as follows:

$$
A(u, v)= \begin{cases}\alpha & \text { if } u=x \text { and } v=y \\ \frac{1-\alpha}{d} & \text { if } u \in \Gamma_{H}(x) \text { and } v-u=y-x \\ 0 & \text { otherwise. }\end{cases}
$$

Observe that $A$ simply moves every mass at vertex $u$ to $v:=u+(y-x)$. Since $y-x \in S, u v$ is always an edge. The cost of this transportation is $\sum_{u, v \in G} A(u, v) d(u, v)=1$. By Equations (2.1) and (2.4), we have $\kappa_{\alpha}(x, y) \geq$ 0 . Thus, $\kappa(x, y) \geq 0$.

Now we show $\kappa(x, y)=0$. We will prove it by contradiction. Suppose $\kappa(x, y)>0$ for some edge $x y \in E(H)$. Let $s:=y-x$ and $\epsilon:=\kappa(x, y)$. We 
have $s \in S$ and $\epsilon>0$. Since $\lim _{\alpha \rightarrow 1} \frac{\kappa_{\alpha}(x, y)}{1-\alpha}=\epsilon$, there exists an $\alpha<1$ such that

$$
\kappa_{\alpha}(x, y)>\frac{\epsilon}{2}(1-\alpha) .
$$

Choose an integer $n>\frac{4}{\epsilon}$. Consider a path $x, x+s, x+2 s, \ldots, x+n s$. For any $g \in G, g$ acts on $H$ as a graph homomorphism. Thus

$$
\kappa(x, y)=\kappa(g+x, g+y) .
$$

In particular, for $1 \leq i \leq n$, we have

$$
\kappa_{\alpha}(x+(i-1) s, x+i s)=\kappa_{\alpha}(x, y)>\frac{\epsilon}{2}(1-\alpha) .
$$

From Equation (2.4), we get

$$
W\left(m_{x+(i-1) s}^{\alpha}, m_{x+i s}^{\alpha}\right)<1-\frac{\epsilon}{2}(1-\alpha) .
$$

From the triangular inequality of the transportation distance, we have

$$
\begin{aligned}
d(x, x+n s)= & W\left(m_{x}^{1}, m_{x+n s}^{1}\right) \\
\leq & W\left(m_{x}^{1}, m_{x}^{\alpha}\right)+\sum_{i=1}^{n} W\left(m_{x+(i-1) s}^{\alpha}, m_{x+i s}^{\alpha}\right) \\
& +W\left(m_{x+n s}^{\alpha}, m_{x+n s}^{1}\right) \\
< & (1-\alpha)+n\left(1-\frac{\epsilon}{2}(1-\alpha)\right)+(1-\alpha) \\
= & n+(1-\alpha)\left(2-\frac{n \epsilon}{2}\right) \\
< & n .
\end{aligned}
$$

In the last step, we apply $2<\frac{n \epsilon}{2}$ by our choice of $n$. Contradiction!

Now we apply Theorem 2 to some special lattice graphs whose vertices are the root lattices in some Euclidean spaces.

Theorem 3. Let $R$ be a root system of type $A_{n}, B_{n}, C_{n}, D_{n}, F_{4}, E_{6}, E_{7}$ or $E_{8}$ (except $G_{2}$ ). Let $G$ be the root lattice, which is viewed as the abelian additive group generated by the roots in $R$. Let $S \subset R$ satisfying $-S=S$. Then the Cayley graph Cayley $(G, S)$ is Ricci-flat.

Proof. Write $H:=$ Cayley $(G, S)$. It suffices to show the conditions of Theorem 2 are satisfied. We only need to verify the following condition: "For any $x \in G$ and $s \in S, d_{H}(x, x+n s)=n . "$ 
By the triangular inequality of the graph distance, we have

$$
d_{H}(x, x+n s) \leq \sum_{i=1}^{n} d_{H}(x+(i-1) s, x+i s) \leq n .
$$

We only need to verify $d_{H}(x, x+n s) \geq n$. We will compare the graph distance to the Euclidean distance as $G$ is embedded as a root lattice. There are two cases.

Case 1. The root system $A_{n}, D_{n}, E_{6}, E_{7}$ and $E_{8}$ have only one type of length, say $l$. For any $x \in G$ and $s \in S$, for any $x, y \in G, d_{H}(x, y)$ be the graph distance while $d(x, y)$ be the Euclidean distance. Then we have

$$
d(x, y) \leq d_{H}(x, y) l
$$

For any $s \in S$ and integer $n$, we have

$$
d_{H}(x, x+n s) \geq \frac{1}{l} d(x, x+n s)=n \frac{d(0, s)}{l}=n .
$$

By Theorem 2, Cayley $(G, S)$ is Ricci-flat.

Case 2. Now we consider the root system of type $B_{n}, C_{n}$ and $F_{4}$. Those root systems have two types of lengths. The ratio of the length of the longer root to one of the shorter root is $\sqrt{2}$. In addition, the angle $\theta$ formed between a longer root $u$ and a shorter root $v$ is either $\frac{\pi}{4}, \frac{\pi}{2}$ or $\frac{3 \pi}{4}$. We have

$$
\langle u, v\rangle=\|u\|\|v\| \cos \theta=\epsilon\|v\|^{2},
$$

where $\epsilon \in\{-1,0,1\}$. Let $l$ denote the length of the shorter root. Then the length of the longer root is $\sqrt{2} l$. Equation (4.3) implies for any shorter root $v$ and any root $u$ (whether short or long)

$$
\langle u, v\rangle \leq l^{2}
$$

If $s$ is a longer root, then we have

$$
d_{H}(x, x+n s) \geq \frac{1}{\sqrt{2} l} d(x, x+n s)=n \frac{d(0, s)}{\sqrt{2} l}=n .
$$

We are done.

If $s$ is a shorter root and $d_{H}(x, x+n s)=k<n$, then we can find $s_{1}, s_{2}, \ldots, s_{k} \in S$ such that $n s=s_{1}+s_{2}+\cdots+s_{k}$. Taking an inner product 


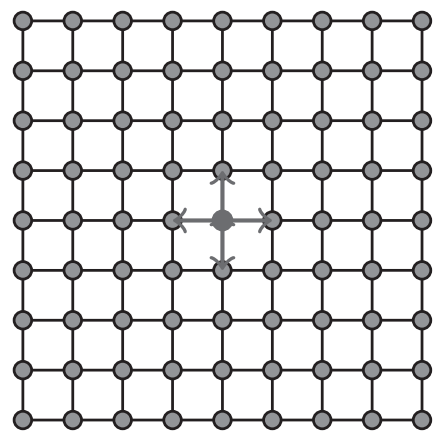

Figure 6: Lattice graph with root system $A_{1}+A_{1}$.

with $s$, we get

$$
n l^{2}=\langle n s, s\rangle=\sum_{i=1}^{k}\left\langle s_{i}, s\right\rangle \leq k l^{2} .
$$

We get $k \geq n$. Contradiction!

By Theorem 2, Cayley $(G, S)$ is always Ricci-flat.

The rank of a root system is the dimension of the underlined Euclidean space. The only rank-1 root system is $A_{1}$, which consists of two roots $\alpha$ and $-\alpha$. The Cayley graph generated by this root system is the infinite path.

The rank-2 root systems $R$ are $A_{2}, B_{2}=C_{2}, D_{2}=A_{1}+A_{1}$ and $G_{2}$. Since $G_{2}$ is excluded from Theorem 3 . Here we get three lattice graphs Cayley $(G, R)$. (See figures 6 to 8 , where the root systems are drawn in red.) We did not get more lattice graphs by taking a subset $S \subseteq R$. For example, for the root system $B_{2}$ and $S$ consisting of three pairs of roots, we get a "skewed" drawing of the graph generated by $A_{2}$. However, for the root system $R$ of rank more than 2 , we do get more Ricci-flat lattice graphs by taking proper subset $S \subsetneq R$.

Combining with the strong covering graph, we get the following corollary.

Corollary 4. Let $H:=$ Cayley $(G, S)$ be the lattice graph constructed in Theorem 3. Let $G^{\prime}$ be a subgroup of $G$ satisfying "any two elements in $G^{\prime}$ has a graph distance at least 6 in $H$." Define the quotient graph $H / G^{\prime}$ with vertex-set $G / G^{\prime}$ and edge-set $u+G^{\prime} \sim v+G^{\prime}$ if $u-v \in S G^{\prime}$. Then $H / G^{\prime}$ is Ricci-flat.

We get many examples of finite Ricci-flat graphs, which can be viewed as the discrete analog of torus. 


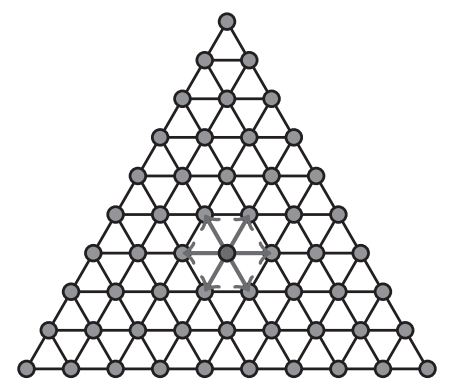

Figure 7: Lattice graph with root system $A_{2}$.

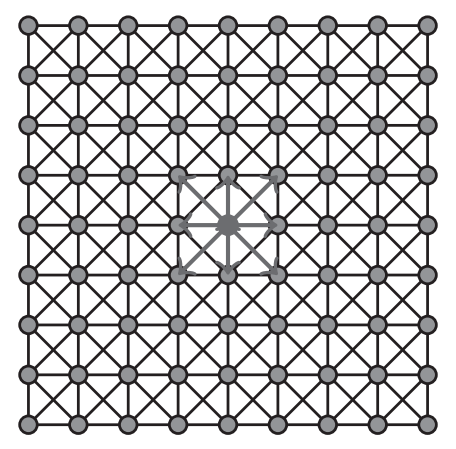

Figure 8: Lattice graph with root system $B_{2}$.

\section{Acknowledgments}

Y.L. was supported by National Natural Science Foundation of China (grant numbers 11271011) and the Fundamental Research Funds for the central universities, and the Research Funds of Renmin University of China (grant numbers 11XNI004). L.L. was supported in part by NSF grant DMS 1000475 and DMS 1300547.

\section{References}

[1] D. Bakry and M. Emery, Diffusions hypercontractives, in 'Séminaire de probabilités', eds. Jacques Azéma and Marc Yor, XIX, 1983/84, Lecture Notes in Math., 1123, Springer, Berlin, 1985, 177-206.

[2] F. Chung and S.-T. Yau, Eigenvalues of graphs and Sobolev inequalities, Combin. Probab. Comput. 4 (1995), 11-25. 
[3] F. Chung and S.-T. Yau, A Harnack inequality for homogeneous graphs and subgraphs, Comm. Anal. Geom. 2 (1994), 627-640, also in Turkish J. Math. 19 (1995), 273-290.

[4] F. Chung, A. Grigor'yan and S.-T. Yau, Upper bounds for eigenvalues of the discrete and continuous Laplace operators, Adv. Math. 117 (1996), $165-178$.

[5] F. Chung and S.-T. Yau, Logarithmic Harnack inequalities, Math. Res. Lett. 3 (1996), 793-812.

[6] F. Chung and S.-T. Yau, A combinatorial trace formula, Tsing Hua lectures on geometry \& analysis (Hsinchu, 1990-1991), International Press, Cambridge, MA, 1997, 107-116.

[7] F. Chung, A. Grigor'yan and S.-T. Yau, Eigenvalues and diameters for manifolds and graphs, Tsing Hua lectures on geometry \& analysis (Hsinchu, 1990-1991), International Press, Cambridge, MA, 1997, 79-105.

[8] F. Chung and S.-T. Yau, Eigenvalue inequalities for graphs and convex subgraphs, Comm. Anal. Geom. 5 (1997), 575-623.

[9] F. Chung and S.-T. Yau, Coverings, heat kernels and spanning trees, Electron. J. Combin. 6 (1999), 21pp.

[10] F. Chung and S.-T. Yau, Spanning trees in subgraphs of lattices, Contemp. Math. 245 (1999), 201-219.

[11] F. Chung and S.-T. Yau, A Harnack inequality for Dirichlet eigenvalues, J. Graph Theory 34 (2000), 247-257.

[12] F. Chung, A. Grigor'yan and S.-T. Yau, Higher eigenvalues and isoperimetric inequalities on Riemannian manifolds and graphs, Comm. Anal. Geom. 8 (2000), 969-1026.

[13] F. Chung and S.-T. Yau, Discrete Green's functions, J. Combin. Theory Ser. A 91 (2000), 191-214.

[14] R. Forman, Bochner's method for cell complexes and combinatorial Ricci curvature, Discrete Comput. Geom. 29(3) (2003), 323-374

[15] Y. Lin, L. Lu and S.-T. Yau, Ricci curvature of graphs, Tohoku Math. J. 63(4) (2011), 605-627. 
[16] Y. Lin and S.-T. Yau, Ricci curvature and eigenvalue estimate on locally finite graphs, Math. Res. Lett. 17 (2010), 345-358.

[17] J. Lott and C. Villani, Ricci curvature for metric-measure spaces via optimal transport, Ann. of Math. (2) 169 (2009), 903-991.

[18] S. Ohta, On measure contraction property of metric measure spaces, Comment. Math. Helv. 82 (2007), 805-828.

[19] Y. Ollivier, Ricci curvature of Markov chains on metric spaces, J. Funct. Anal. 256(3) (2009), 810-864.

[20] K.-T. Sturm, On the geometry of metric measure spaces, (I),(II), Acta Math. 196 (2006), 65-131, 133-177.

Renmin University of China

BEIJING 100872

China

E-mail address: linyong01@ruc.edu.cn

University of South CAROLINA

Columbia, SC 29208

USA

E-mail address: lu@math.sc.edu

HARVARD UNIVERSITY

CAMBridge, MA 02138

USA

E-mail address: yau@math.harvard.edu

ReCeived JAnuARY 2, 2013 
